\title{
An Emergence of a MRAB: With Growing Necessity of Antibiotic Pharmacist in Infectious Era
}

\author{
Maria A*, Somia G, Hina L, Ishrat I and Munawerah F \\ Faculty of Pharmacy, Jinnah University for Women, Karachi, Pakistan
}

*Corresponding author: Maria A, Lecturer, Faculty of Pharmacy, Jinnah University for Women, Karachi 74600, Pakistan, Tel: +92331-3119160; E-mail: maria.ayub2000@gmail.com

Received date: May 17, 2016; Accepted date: Jun 13, 2016; Published date: Jun 17, 2016

Copyright: ( 2016 Maria A, et al. This is an open-access article distributed under the terms of the Creative Commons Attribution License, which permits unrestricted use, distribution, and reproduction in any medium, provided the original author and source are credited.

\begin{abstract}
Multi-drug Resistant pathogens pose a serious challenge against lifesaving antimicrobials globally. MRAB (multi drug resistant Acinetobacter baumannii) has a capability for extensive adaptation of multi-drug resistance pattern particularly in immune compromised patients which became resistant to carbapenems beta lactamase antimicrobials. The objective of the study is to evaluate multidrug resistance pattern of $A$. baumannii among broad spectrum antimicrobials with intention to highlight the growing need of antibiotic pharmacist in south Asia to encounter such life threatening multidrug resistance pattern. For the purpose of this study MRAB isolates with sensitivity and resistance pattern carefully evaluated by hospital pharmacist to determine such pattern with rationale utilization of therapy. Resulting data of about 146 isolates of MRAB interpreted by pharmacist which shows about $78 \%$ isolates were resistant to more than four broad spectrum antimicrobials. Amoxicillin has been resistant to $90 \%$, Piperacillin $67 \%$, third generation cephalosporin $92 \%$ with $75 \%$ of fourth generation cefepime, carbapenems $67 \%$, aminoglycosides $75 \%$, quinolones $54 \%$ and Polymyxin B $47 \%$ resistance respectively. This retrospective studies drawn conclusion that the effective utilization with evaluation of adaptive drug resistance can encounter threats associated with multidrug resistance pathogens and pharmacist can imparts key role in rationale utilization of antimicrobials with growing concern of implementation of specialty role in healthcare sector of south Asia.
\end{abstract}

Keywords: MRAB; Evaluation; Pathogenesis; Drug resistance; Sensitivity

\section{Introduction}

Multi-drug Resistant pathogens create an emergence against life threatening antimicrobials is a major growing concern and challenging task Globally and in the USA [1-2]. Regardless of this serious issue with increase in threshold of antimicrobials resistance, termination of development of new antimicrobials by pharmaceutical industries create an emergence to treat multidrug resistant pathogens [3-6]. However the adaptation of pathogens resistance against wide range of antimicrobials among gram positive and gram negative microbes Acinetobacter baumannii, Klebsiella pneumonia, Pseudomonas aeruginosa, Klebsiella pneumonia, MRSA, VRSA and E. coli emerge a serious concern to encounter infectious disease [7].

A. baumannii, a superbug with gram negative staining aerobe is versatile pathogen having high threshold among immunodeficient patients predominantly exposed to prolong hospital admissions [8]. Acinetobacter accounts for significant taxonomic variation globally resistant to variety of antimicrobial is a major growing concern for Health sector and create emergence against broad spectrum antimicrobials. This pathogen have prolong survival threshold especially measure leading cause of hospital acquired nosocomial infections. It targets the open skin wounds and respiratory tract infection and mucosal secretions, pneumonia in hospital setting mostly caused by this pathogen additionally it affects bone, nervous system and infection involving soft tissues is major growing concern in hospital settings [8-10].
This pathogen presented as high alert with red alarming situation due to multidrug resistant antimicrobial spectrum $[8,11]$. Acinetobacter, has taken importance globally in term of current and scientific modified era day by day and reviewed previously in 19th century [12] incidence of multidrug resistance microbes presently is serious threat for both community and hospital acquired nosocomial infection the prevalence of antimicrobial resistance accounts for the 3rd major leading concern in health sector by world Health organization (WHO). Klebsiella pneumoniae, A. baumannii, Staphylococcus aureus, Enterococcus faecium, Pseudomonas aeruginosa classified as multidrug resistant pathogens $[8,13]$.

A. baumannii accounts for meningitis, soft skin sepsis, urinary tract infections, bronchitis and endocarditis presenting a challenging concern primarily in the intensive care units [14]. Infection resulting from this pathogen often hard due to highly acquired resistance mechanism by this bad bug especially beta-lactamase inducing resistance mechanism of in gram negative aerobe $[14,15]$. Although $A$. baumannii reported in past 1970s possess sensitivity against variety of antimicrobials but now in current era of infectious disease $A$. baumannii have adapted a resistance mechanism against first line antimicrobials [16,17]. Pathogenicity caused by this pathogens specially targets soft tissue open wounds exposed as a result of accidental injury and burns and mucosal lining of respiratory tract $[8,10,17,18]$. Although, $A$. baumannii accounts rarely found as normal flora of skin approximately just $3 \%$ according to one study in spite of the this fact its responsible for skin infection [19]. A. baumannii is isolated mostly from people about $22 \%$ in homeless serve as host for this pathogen [20]. A. baumannii threshold is high among soldier infected with open wounds infections [21]. 
A. baumannii creates an emergence once it has been isolated in any healthcare setting due to high risk pathogenicity especially in the intensive care units patient with severe illness [22]. People with immunodeficiency with prolonged stay at hospital are highly at risk infected with this pathogens. People exposed to ventilating devices and other disposable devices present high risk group susceptible the infection associated with baumannii due to its ability to make biofilm which colonize mucosal lining of respiratory tract [23-25]. Ability of $A$. baumannii to adapt extensive and multi-drug resistance according to environmental factor imparts a contributing factor for emergence of resistance with acquisition of Innate adaptive resistance mechanism by up regulation considerably taken into account as multidrug resistance A. baumannii MRAB with rapid emergent resistant species of $A$. baumannii resistant with beta-lactams drugs even with carbapenems [26-28].

For the improvement of rationale utilization of drug therapy role of pharmacist must be evident to utilize properly of available drugs and the growing need for pharmacists does exist in the clinical area proved by the study conducted at California San Francisco medical center proven the role of pharmacist additionally to the medical staff and nursing staff. New drug formulary system to elaborate the role of pharmacist in patient care and the clinical pharmacist can play key role for drug information resurgence and propagate role in healthcare setting effectively [29]. Extensive irrational prescribing threshold of antimicrobials in hospital settings at UK, the role of introduction of antibiotic pharmacist specialty with proper monitoring of antibiotic utilization, its indication by clinicians, and provide sound knowledge of antimicrobials to all healthcare co-providers. Pharmacist should need to prove their role in drug intervention may imparts their beneficial role in many situations to expand their role by taking effective decision such as antimicrobial stop and shifting towards intravenous route to oral safe route and other important interventions as necessary to augment patient care [30].

The objective of this study was to identify the multidrug resistance pattern against lifesaving antimicrobials by their sensitivity and resistance pattern against $A$. baumannii with growing concern that the need of antibiotic pharmacist in Pakistan healthcare sectors and to find out the target rational therapy to encounter the multidrug resistance threaten associated with irrational practice in healthcare sector and clinical pharmacist may play a key role in appropriate utilization of this antimicrobials against bad bug pathogens. Multidrug resistance is a major growing concern in hospital setting especially in the intensive care units of public and private health care sector primarily in immune compromised patients.

\section{Methodology}

\section{Collection of bacterial strains}

About 146 clinical isolates of Acinetobacter baumannii resistant to multiple drugs ( $90 \%$ to penicillin \& $75 \%$ to cefepime) were obtained from different secondary and tertiary care center of Karachi of public and private sector. Isolates obtained from in and out patients were mostly from chronically ill and immune compromised system and those admitted at intensive care units. Specimen collection from these source specifically designed in this study to evaluate multidrug resistance $A$. baumannii.

Isolates were tested in the laboratories on the basis of their biochemical testing and colony growth characteristics and finally preserved on slant at controlled temperature of $4^{\circ} \mathrm{C}$. Antimicrobial susceptibility and resistance evaluated by according to CLSI Reference standard of Clinical and laboratory standard institute.

\section{Preparation of medium \& its innoculation}

Antibiograms obtained from all participant healthcare centers were based on Kirby-Bauer Disk diffusion method and inoculums of micro broth dilutions prepared by Muller-Hinton broth (MHB) with suspended colonies were equivalent to $0.5 \mathrm{McF}$ arland Standard. All results interpreted on sheets complied according to CLSI (NCCLS) [31]. Incubation period for this method is $35 \pm 2{ }^{\circ} \mathrm{C}$ with supportive ambient air for 20-24 hours.

\section{Susceptibility testing}

More than 35 antibiotics were selected against $75 \%$ cefepime and $90 \%$ resistant isolates of $A$. baumannii with Disc content of antimicrobial piperacillin $100 \mu \mathrm{g}$, Cefepime \& Ceftazidime $30 \mu \mathrm{g}$, Monobactams (Aztreonam) $30 \mu \mathrm{g}$, meropenem \& imipenem $10 \mu \mathrm{g}$, Aminoglycosides (Amikacin) $30 \mu \mathrm{g}$, (tobramycin and gentamycin) 10 $\mu \mathrm{g}$ and quinolones (ciprofloxacin) $5 \mu \mathrm{g}$ correspondingly according to CLSI directed zone of resistance and susceptibility size with exclusion of intermediate size zone.

\section{Incorporation of pharmacist}

After the collection of testing report of 4th Generation resistant isolate $A$. baumannii Pharmacist paid their attention and presented detailed report on rationale utilization of antibiotics upon invitation by healthcare authorities and they introduced key points regarding proper utilization of antimicrobials and to encounter resistance associated with Multi drug resistant pathogens.

They can designed and improve their hospital formulary according to patient susceptibility response in view of the fact to make sure rational utilization of antimicrobials can prevent this major issue against Bad bugs after reported linezolid and glycopeptides resistance in tertiary care centers. Resulting outcome analyzed statistically by applying IBM SPSS Statistics 21 version software and p-value less than 0.05 considered significant statistically.

\section{Results}

After careful evaluation with hospitals Antibiograms of MRAB All resulting data interpreted by hospital pharmacist with the help of statistical analysis and spss software to provide key pattern of drug resistance adaptive mechanism in MRAB isolates.

For the measurement of resulting outcomes of antimicrobials sensitivity pattern more than 146 isolates were evaluated from January 2013-august 2015 from different tertiary healthcare center of patient with chronic illness.

Samples collection source were mostly from blood, urine, pus and sputum with highest percentage of blood sample shown in Figure 1. 
Citation: Maria A, Somia G, Hina L, Ishrat I, Munawerah F (2016) An Emergence of a MRAB: With Growing Necessity of Antibiotic Pharmacist in

Page 3 of 6

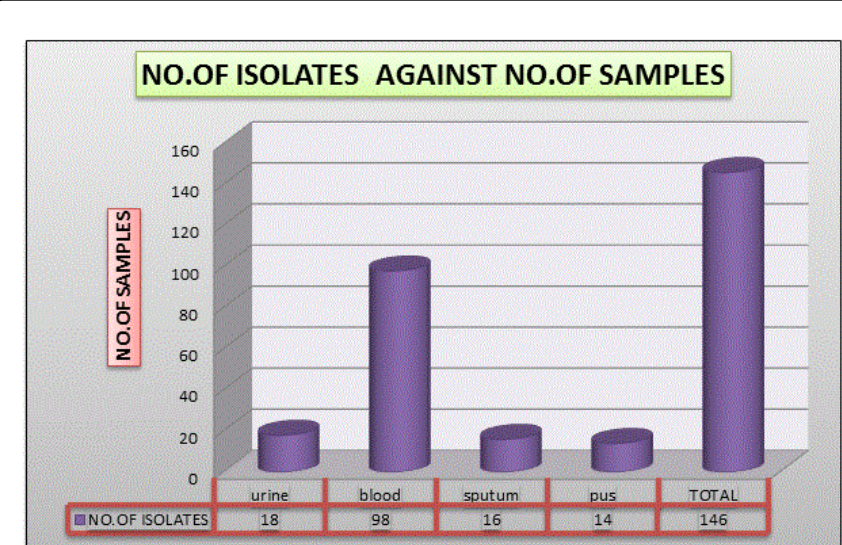

Figure 1: No of samples of isolated species of A. baumannii.

All the isolates were tested against more than 20 antimicrobials and the resulting Antibiograms clearly shows that about $78 \%$ isolates were resistant to more than four broad spectrum antimicrobials shown in Figure 2.

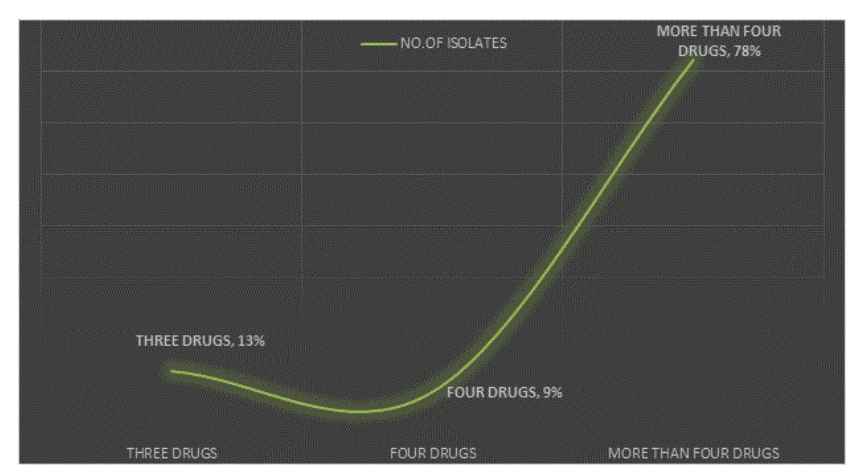

Figure 2: Percentage of isolate resistance against three, four and multiple drugs.

Resulting Antibiograms of MRAB from different tertiary care center were $75 \%$ resistant to 4 th generation cephalosporins which clearly reflects less efficacy of beta lactam antibiotics Figure 3 clearly demonstrate $86 \%$ resistance against piperacillin, $82 \%$ cefepime, carbapenems 79\%, aminoglycosides $88 \%$, quinolones $95 \%$ and Polymyxin-B $94 \%$ respectively in isolates of MRAB resistant to three broad spectrum antimicrobials.

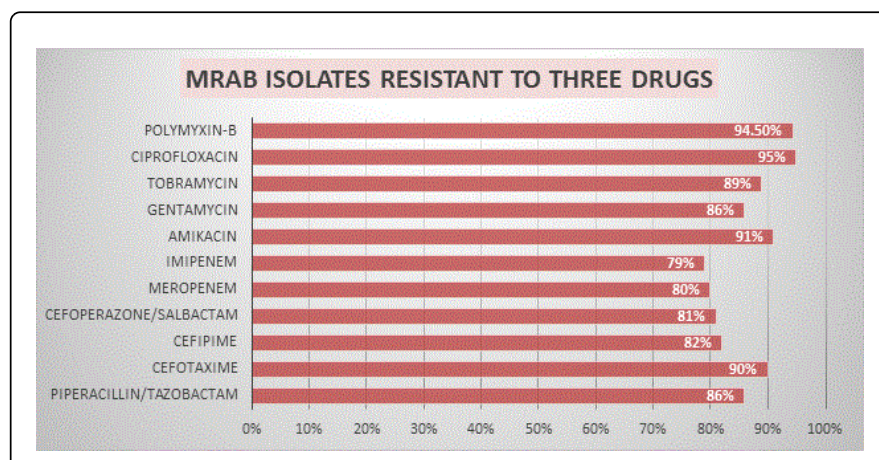

Figure 3: Drug resistance pattern of MRAB resistant to three antimicrobials.

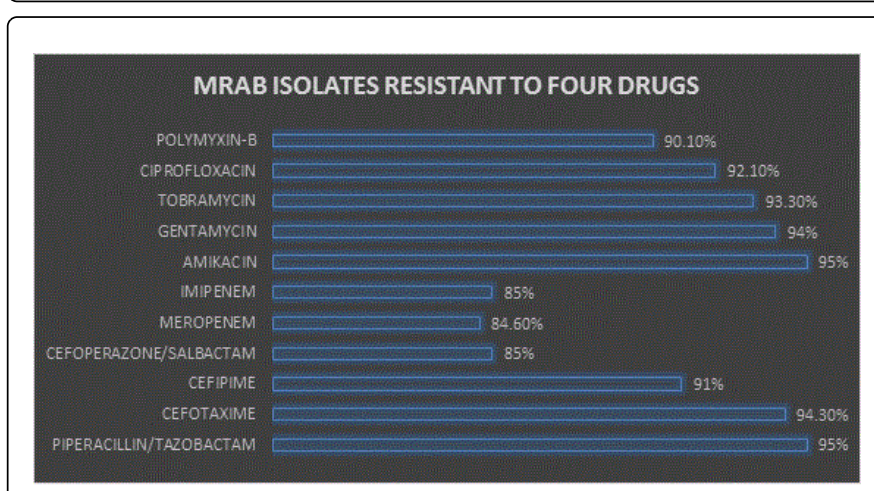

Figure 4: Drug resistance pattern of MRAB resistant to four antimicrobials.

Figure 4 and Table 1 shows isolates which were resistant to four drugs involving loss of sensitivity in amikacin \& piperacillin 95\%, quinolones $92 \%$, cefepime $91 \%$ with highest sensitivity shown in carbapenems of $15 \%$ respectively. From T-test the resulting outcome is significant which clearly reveals the fact that there is significant change in sensitivity and resistance pattern among different class of antimicrobials $(\mathrm{p}<0.05)$.

\begin{tabular}{|l|l|l|}
\hline Parameter & Chloroform Extract & Acetogenin fraction \\
\hline Total soluble polyphenols $(\mathrm{mg} / \mathrm{g} \mathrm{DW})$ & $0.72 \pm 0.02$ & $0.032 \pm 0.001$ \\
\hline ABTS assay (mmol TE/g DW) & $22.19 \pm 0.15$ & $4.91 \pm 0.23$ \\
\hline DPPH assay (mmol TE/g DW) & $1.02 \pm 0.14$ & $0.58 \pm 0.07$ \\
\hline Reducing power $(\mu \mathrm{M} \mathrm{AA} \mathrm{equivalent)}$ & $23.85 \pm 0.07$ & $21.77 \pm 0.39$ \\
\hline Total antioxidant activity $(\mu \mathrm{g}$ a-tocopherol equivalent) & $33.76 \pm 2.91$ & $4.85 \pm 2.10$ \\
\hline
\end{tabular}

Table 1: Total soluble polyphenols and antioxidant activity of chloroform extract and acetogenin fraction obtained from Annona muricata fruit pulp. 


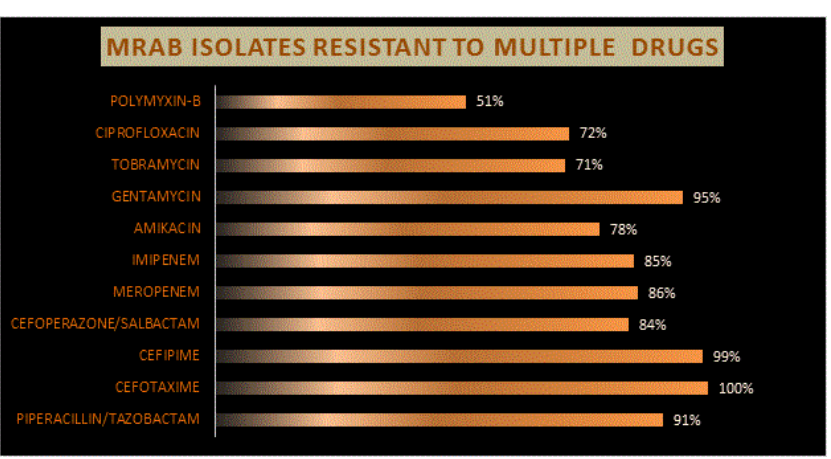

Figure 5: Drug resistance pattern of MRAB resistant to multiple antimicrobials.

Multiple drug resistant isolates shows highest sensitivity against Polymyxin-B 49\%, ciprofloxacin 30\%, aminoglycosides $27 \%$, carbapenems $15 \%$, with highest percentage of resistance against cephalosporins about 99\% respectively shown in Figures 5 and 6.

Table 1 and Figure 6 demonstrate comparative efficacy with less sensitivity of antimicrobials against MRAB and pharmacist evaluated these all MRAB evolution of resistance against broad spectrum antimicrobials which provide best treatment option with less chance of resistance particularly in immune compromised patients.

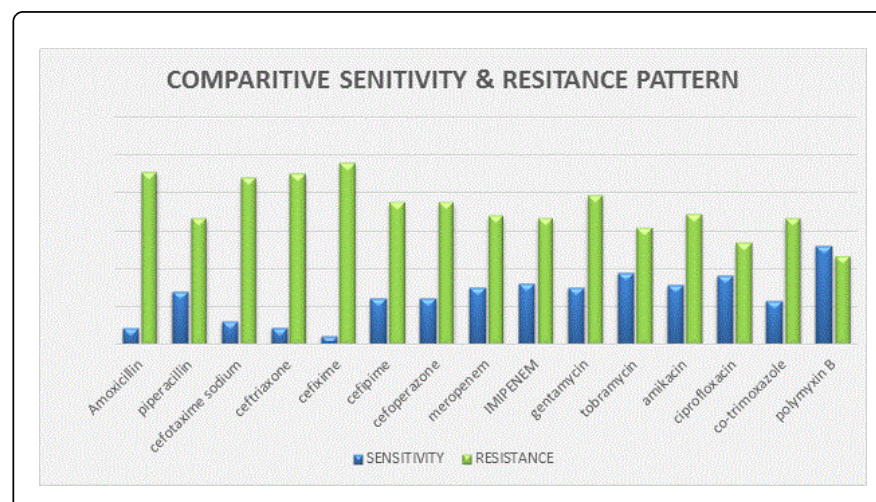

Figure 6: Shows comparative efficacy against MRAB.

\section{Discussion}

Contingency of strains that are resistant to nearly all available drugs and receivable to increase in antimicrobial resistance, Acinetobacter baumannii is attracting much recognition. All of the considerable resistance mechanisms that are known to fall in bacteria, the resistance of $A$. baumannii to antimicrobial agents is mediated also through this mechanism involving target sites adaptation, enzymes inactivation, drugs ornament of decreased influx as well as increased ornament of active efflux. Associated with the resistance beta lactamases is the most sundry group of enzyme and have been distinguished so far in $A$. baumannii of type of this enzyme is about more than 50 enzymes. At least nine definite modifying enzymes by the acknowledgment in some strains of the Aminoglycosides resistance [32]. Resistant to the agents implicated Aminoglycosides, beta lactamase as well as fluoroquinolones in strains of $A$. baumannii an upward mode have proposed by several studies. Among clinicians, the resistance amidst $A$. baumannii strains to $\beta$-lactam agents is of great interest. This study clearly illustrates and identified the sources of MRAB in terms of isolation which about $98 \%$ from the blood. In this study, spectrum of antibiotic is assessed with especial attention of pharmacist and shows multiple drug resistance species of species against more than four antimicrobials up to $78 \%$ resulting an emergence condition.

This study also demonstrated the pattern of multi drug resistance against the broad spectrum antimicrobials like carbapenems class from which MRAB resist up to $80 \%$ from imipenem, a novel drug in this modern era so creating a difficult situation for health care partitions as highlighted by involvement of pharmacist. Well addition to this resistance which is very important concern in healthcare settings, treatment of this multidrug resistance pathogen cannot also be avoided. Treatment against this pathogen is a key point in this study effectively evaluated by the involvement of the pharmacist as MRAB resistance most commonly found in immune compromised patients, critically ill patients such as who admitted in ICU, so in these conditions choice of antibiotic should be rationale to provide maximum therapeutic effect as the necessity of these type of patients. Antimicrobials sensitivity against MRAB, from which higher percentage from Polymyxin B obtained up to $49 \%$ later this $27 \%$ sensitivity obtained from aminoglycosides and the hallmark of the antibiotic era is that MRAB is sensitive to carbapenems is only about $15 \%$ which is the novel drug in clinical setting now a days. In this study pharmacist also highlighted the main concern of antimicrobials relative to clinical point of view that now a days cephalosporins are commonly use against many infections in terms of drug of choice in irrational therapy but MRAB resistance is found up to $99 \%$ against cephalosporins. In $A$. baumannii resistance to extended-spectrum cephalosporins the main mechanism involved are the plasmidencoded Ambler class A, B, and D $\beta$-lactamases and cephalosporins chromosomal over-expression [33].

From various geographic areas hospitals epidemics have been outlined about MRAB [34-37]. A. baumannii is able to grow at numerous temperature conditions and $\mathrm{PH}$ as well as it does not have any fastidious growth requirements [38]. To persist in either moist or dry conditions in the hospital environment contributing transmission The all-around organism exploits a variety of both carbon and energy sources and this characteristics explains the ability of Acinetobacter species $[39,40]$. Although it is prevalent in hospitals and rare isolates have been found in community suggested by literature review but recently some community cases of this pathogen has also reported. In northern Australia and southern Asia an occurrence of communityacquired was recently described associated with high mortality in patients. In that study were bacteremia (31.6\%) and had acute respiratory distress syndrome and disseminated intravascular coagulation with a significant percentage of the patients [41-43]. We will not be able to use anymore antimicrobial agents against this opportunistic pathogen among beta lactams antibiotics as well as we need to used tool having ability to sort out the resistance mechanism and there is need of discovery of antimicrobials which could treat effectively this isolate. Infection control is also can be used to control resistance by imparting various health workers like pharmacists.

Pharmacist are integral part of healthcare team their specialty role i.e., antibiotic Pharmacist concept is still controversial in south Asia as patients need no suggestion for safe utilization of antimicrobials other than physician. Pharmacist can utilize their knowledge regarding rational utilization of drug to prevent this life threatening resistance 
against infectious disease. In this study Pharmacist from different hospital setting make their effort to designed strategy to combat antimicrobial resistance. Pharmacist better suggestion make their necessity in incorporation of major health issues which is accepted by healthcare practitioner and high authorities as they suggested incorporation of antibiotic pharmacist in south Asia is now obligatory. It is not an effortless assignment in $A$. baumannii with a fulfillment of multidrug resistance. By the consequences, new antimicrobial which regulate this pathogen there is also a decided downturn in the advancement.

The end of the "antibiotic era" may be faced soon by us. The fruit of human infinite, has been responded by an escalation of resistance mechanisms in bacteria because the initial and seemingly unstoppable success of antibiotics.

\section{Conclusion}

An emergence of Multidrug resistant Acinetobacter baumannii create an emergence against lifesaving antimicrobials because of loss of sensitivity in infectious era. Patient with chronic illness need rational utilization of antimicrobials to combat resistance against superbug. Inclusion of antibiotic pharmacist can encounter this problem in south Asia as essential lacking of evident role of pharmacist in specialty care in immune deficient patients. Pharmacist have useful knowledge regarding drug selection, utilization, monitoring of adverse drug reaction and drug effectiveness against infectious disease. Their inclusion in health care team against infectious disease can minimize resistance and loss of antimicrobial efficacy by finding bacterial evolution mechanism.

\section{References}

1. Smolinski MS, Hamburg MA, Lederberg J (2003) Microbial Threats to Health: Emergence, Detection, and response. Washington, DC: The Institute of Medicine.

2. Diekema DJ, Bootsmiller BJ, Vaughn TE, Woolson RF, Yankey JW, et al. (2004) Antimicrobial Resistance Trends and Out-Break Frequency. Clin Infect Dis 38: 78-85.

3. Spellberg B, Powers JH, Brass EP, Miller LG, Edwards JE Jr (2004) Trends In Antimicrobial Drug Development: Implications Forthefuture. Clin Infect Dis 38: 1279-1286.

4. Norrby SR, Nord CE, Finch R (2005) Lack of development of new antimicrobial drugs: a potential serious threat to public health. Lancet Infect Dis 5: 115-119.

5. Wenzel RP (2004) The antibiotic pipeline--challenges, costs, and values. N Engl J Med 351: 523-526.

6. DeMaria A Jr (2005) Challenges of sexually transmitted disease prevention and control: no magic bullet, but some bullets would still be appreciated. Clin Infect Dis 41: 804-807.

7. Lister PD, Wolter DJ, Hanson ND (2009) Antibacterial-Resistant Pseudomonas Aeruginosa: Clinical Impact And Complex Regulation of Chromosomally Encoded Resistance Mechanisms. Clin Microbiol Rev 22: 582-610.

8. Howard A, O'Donoghue M, Feeney A, Sleator RD (2012) Acinetobacter baumannii: An Emerging Opportunistic Pathogen. Virulence 3: 243-250

9. Song JY, Cheong HJ, Choi WS, Heo JY, Noh JY, et al. (2011) Clinical and microbiological characterization of carbapenem-resistant Acinetobacter baumannii bloodstream infections. J Med Microbiol 60: 605-611.

10. Kempf M, Eveillard M, Deshayes C, Ghamrawi S, Lefrançois C, et al. (2012) Cell surface properties of two differently virulent strains of Acinetobacter baumannii isolated from a patient. Can J Microbiol 58: 311-317.
11. Jeters R, Sóki J (2013) IS Elements In Antibiotic-Resistant Bacteroides. WJCID 3: 8 .

12. Kurcik-Trajkovska B (2009) Acinetobacter Spp.-A Serious Enemy Threatening Hospitals Worldwide. Macedonian Journal of Medical Sciences 2: 157-162.

13. Gülmez D (2013) Bakterilerde Saptanan Yeni Direnç Mekanizmalarinin Yansimalari. Ankem Derg 27: 158-166.

14. Go ES, Urban C, Burns J, Kreiswirth B, Eisner W, et al (1994) Clinical and molecular epidemiology of acinetobacter infections sensitive only to polymyxin B and sulbactam. The Lancet 344: 1329-1332

15. Chastre J, Trouillet JL (2000) Problem Pathogens (Pseudomonas Aeruginosa and Acinetobacter). Semin Respir Infect 15: 287-298.

16. Huang XZ, Cash DM, Chahine MA, Nikolich MP, Craft DW, et al. (2012) Development And Validation Of A Multiplex Taqman Real-Time PCR for Rapid Detection of Genes Encoding Four Types of Class D Carbapenemase In Acinetobacter baumannii. J Med Microbiol 61: 1532-1537.

17. Wand ME, Bock LJ, Turton JF, Nugent PG, Sutton JM (2012) Acinetobacter baumannii Virulence Is Enhanced In Galleria Mellonella Following Biofilm Adaptation. Journal of Medical Microbiology 61: 470-477.

18. Lin MF, Lan CY Antimicrobial Resistance In Acinetobacter baumannii, World Journal of Clinical Cases ESPS.

19. Seifert H, Dijkshoorn L, Gerner-Smidt P, Pelzer N, Tjernberg I (1997) Distribution of Acinetobacter Species on Human Skin: Comparison of Phenotypic and Genotypic Identification Methods. J Clin Microbiol 35: 2819-2825.

20. La Scola B, Raoult D (2004) Acinetobacter baumannii in Human Body Louse. Emerg Infect Dis 10: 1671-1673.

21. (2004) Acinetobacter baumannii Infections Among Patients At Military Medical Facilities Treating Injured U.S. Service Members, MMWR, 53: 1063-1066.

22. Montefour K, Frieden J, Hurst S, Helmich C, Headley D, et al. (2008) Acinetobacter baumannii: an emerging multidrug-resistant pathogen in critical care. Crit Care Nurse 28: 15-25.

23. Bayuga S, Zeana C, Sahni J, Della-Latta P, El-Sadr W (2002) Prevalence and antimicrobial patterns of Acinetobacter baumannii on hands and nares of hospital personnel and patients: the iceberg phenomenon again. Heart Lung 31: 382-390.

24. Gusten WM, Hansen EA, Cunha BA (2002) Acinetobacter baumannii Pseudomeningitis. Heart Lung 31: 76-78.

25. Lorente C, Del Castillo Y, Rello J (2002) Prevention of infection in the intensive care unit: Current advances and opportunities for the future. Curr Opin Crit Care 8: 461-464.

26. Peleg AY, Seifert H, Paterson DL (2008) Acinetobacter baumannii: Emergence of a Successful Pathogen. Clin Microbiol Rev 21: 538-582.

27. Vaneechoutte M, Young DM, Ornston LN, De Baere T, Nemec A, et al. (2006) Naturally Transformable Acinetobacter Sp. Strain ADP1 Belongs To The Newly Described Species Acinetobacter Baylyi. Appl Environ Microbiol 72: 932-936.

28. Bacher JM, Metzgar D, de Crécy-Lagard V (2006) Rapid evolution of diminished transformability in Acinetobacter baylyi. J Bacteriol 188: 8534-8542.

29. William ES (2007) Role of a Pharmacist in Improving Rational Drug Therapy as Part of the Patient Care Team, Annals of Pharmacotherapy 41: 330-335.

30. Weller TMA, Jamieson CE (2004) The expanding role of the antibiotic pharmacist, Journal of Antimicrobial Chemo 54: 295-298.

31. National Committee for Clinical Laboratory Standards (NCCLS) (2003) Performance Standards for Antimicrobial Susceptibility Testing. NCCLS Approved Standard M100-S10. Wayne, PA.

32. Seward RJ, Lambert T, Towner KJ (1998) Molecular Epidemiology of Aminoglycoside Resistance in Acinetobacter Spp. J Med Microbiol 47: 455-462. 
Citation: Maria A, Somia G, Hina L, Ishrat I, Munawerah F (2016) An Emergence of a MRAB: With Growing Necessity of Antibiotic Pharmacist in Infectious Era. Pharm Anal Acta 7: 491. doi:10.4172/2153-2435.1000491

Page 6 of 6

33. Nemec A, Janda L, Melter O, Dijkshoorn L (1999) Genotypic and phenotypic similarity of multiresistant Acinetobacter baumannii isolates in the Czech Republic. J Med Microbiol 48: 287-296.

34. Acar JF (1997) Consequences of bacterial resistance to antibiotics in medical practice. Clin Infect Dis 24: S17-S18.

35. Ayan M, Durmaz R, Aktas E, Durmaz B (2003) Bacteriological, clinical and epidemiological characteristics of hospital-acquired Acinetobacterbaumannii infection in a teaching hospital. J Hosp Infect 54: $39-45$.

36. Bergogne-Berezin E, Towner KJ (1996) Acinetobacter spp. as nosocomia pathogens: microbiological, clinical, and epidemiological features. Clin Microbiol Rev 9: 148-165.

37. Landman D, Quale JM, Mayorga D, Adedeji A, Vangala K, et al. (2002) Citywide clonal outbreak of multiresistant Acinetobacter baumannii and Pseudomonas aeruginosa in Brooklyn, NY: the preantibiotic era has returned. Arch Intern Med 162: 1515-1520.

38. Bergogne-Berezin E, Towner KJ (1996) Nosocomial pathogens: microbiological, clinical, and epidemiological features. Clin Microbiol Rev 9: 148-165.
39. Getchell-White SI, Donowitz LG, Groschel DH (1989) The inanimate environment of an intensive care unit as a potential source of nosocomial bacteria: evidence for long survival of Acinetobacter calcoaceticus. Infect Control Hosp Epidemiol 10: 402-407.

40. Wendt C, Dietze B, Dietz E, Rüden H (1997) Survival of Acinetobacter baumannii on dry surfaces. J Clin Microbiol 35: 1394-1397.

41. Chen MZ, Hsueh PR, Lee LN, Yu CJ, Yang PC, et al. (2001) Severe community-acquired pneumonia due to Acinetobacter baumannii. Chest 120: 1072-1077.

42. Leung WS, Chu CM, Tsang KY, Lo FH, Lo KF, et al. (2006) Fulminant community-acquired Acinetobacter baumannii pneumonia as a distinct clinical syndrome. Chest 129: 102-109.

43. Falagas ME, Karveli EA, Kelesidis I, Kelesidis T (2003) Communityacquired Acinetobacter baumannii pneumonia. Rev Clin Esp 203: 284-286. 